\title{
Consumer response to car fronts: eliciting biological preparedness with product design
}

\author{
Christian Purucker • David E. Sprott • \\ Andreas Herrmann
}

Received: 20 September 2012/ Accepted: 14 November 2013/Published online: 6 December 2013

(C) Springer-Verlag Berlin Heidelberg 2013

\begin{abstract}
Building on assumptions derived from evolutionary theory, we investigated viewers' reactions to the design of car fronts (i.e., an automobile's face), which were designed to be threatening using basic principles of anthropomorphism. Previous research suggests two opposite human reactions when presented with threatening stimuli: Initially, threatening objects attract human attention (e.g., when exploring a scene for the first time), but afterwards, people tend to avoid such threatening stimuli (as they are likely to induce discomfort in the viewer). This proposition is tested within a product design context using eye tracking methodology. Results showed that automotive stimuli not only activate affective dimensions of customers, but also lead to specific automatic reactions that can be explained by evolutionary theory. Practical implications for product design and marketing are discussed.
\end{abstract}

Keywords Anthropomorphism - Product design - Eye tracking ·

Biological preparedness

JEL Classification $\mathrm{M} 19 \cdot \mathrm{M} 30 \cdot \mathrm{M} 31 \cdot \mathrm{L62}$

C. Purucker

Department of Psychology III, University of Würzburg, Würzburg, Germany

e-mail: Christian.Purucker@uni-wuerzburg.de

D. E. Sprott

College of Business, Washington State University, Pullman, WA, USA

e-mail: dsprott@wsu.edu

A. Herrmann (ه)

Center for Customer Insight, University of St. Gallen, St. Gallen, Switzerland

e-mail: Andreas.Herrmann@unisg.ch 


\section{Introduction}

"Did that car stare at me like a person? And if it did, what affective expressions did the car's face display? Was it being aggressive or was it smiling?" As exemplified in fictitious movies like "The Love Bug" from 1969, featuring a talking VW Beetle named "Herbie," children and grown-ups alike have considered the human characteristics possessed by products used in everyday life. Prior research clearly indicates that people often ascribe animistic or even anthropomorphic (human-like) properties to non-living objects (e.g., Aggarwal and McGill 2007; Epley et al. 2007; Waytz et al. 2010). Not surprisingly, designers and product managers are willing to consider dimensions of the product's human nature (DiSalvo and Gemperle 2003). Indeed, the elicitation of anthropomorphic thoughts may help establish closer customer bonds, trigger positive emotional associations and create a clearer differentiation from competitive products (cf. Aggarwal and McGill 2007).

One product category wherein anthropomorphic design can be usefully applied is the automobile, particularly with regard to the car's face (or front; Landwehr et al. 2011), where at least $90 \%$ of participants in a recent study associated animate properties to real-life car fronts (Windhager et al. 2008). Other research shows that a car's face can elicit a wide range of consumer reactions from an anthropomorphic design (cf. Miesler et al. 2011). For example, car fronts could evoke conscious attribute associations (e.g., "the car looks cute and vulnerable") or elicit semiconscious or even unconscious affective reactions (e.g., "I think this car looks dynamic, but maybe it looks a little too aggressive").

While prior research has considered whether anthropomorphic automotive designs can elicit reactions similar to responses to human faces (e.g., Windhager et al. 2010; Miesler et al. 2010), prior empirical studies have mainly focused on subjective ratings or behavioral responses that can be consciously controlled by research participants. Basic research on ethnology and developmental psychology suggests that reactions to schematic faces might not solely exist at a cognitive level, however, but rather involve motoric reactions like involuntary and reflectory approach or avoidance responses (cf. Lorenz 1950). Such evolutionary mechanisms would primarily show up in a person's unconscious reactions and behavioral patterns and only subsequently in conscious judgment and verbal responses.

Therefore, in the current paper, we explore whether a car face creates automatic responses similar to those elicited by a human face. In particular, prior research in psychology has demonstrated an advantage for processing threatening faces (e.g., these stimuli can be detected faster in a crowd of other faces) that is supportive of an evolutionary mechanism for face perception (e.g., Öhman et al. 2001). Assuming similar mechanisms for anthropomorphic product designs, we should be able to demonstrate similar findings with aggressive automobile faces. In two eye-tracking studies, we show that anthropomorphized car fronts manifest specific behavioral and attentional effects that are dependent upon the affective value they promote. These effects suggest that anthropomorphic product design can be employed to capture customer's attention beyond what can be readily assessed by liking ratings. We discuss our findings in terms of future research directions and practical implications for product designers. 


\section{Theoretical background}

\subsection{Anthropomorphism in product design}

Although product design has long been known to drive market success, the role of design in product-related outcomes had been traditionally neglected in the marketing literature. Bloch's (1995) model changed this situation by providing insights into the relationship between a product's design and consumer's direct psychological responses, as well as distal behavioral reactions. Building on these ideas, anthropomorphism in product design has been recently introduced to the marketing literature by researchers investigating how consumers anthropomorphize products in the presence of design characteristics congruent with associated human schemata (e.g., Aggarwal and McGill 2007). This research provides clear evidence that humans can and often do anthropomorphize products and that some designs are perceived in a way comparable to the human analog. While much of this work has been demonstrated with anthropomorphic product faces in the field of car design, findings have also been confirmed for other categories, such as beverage bottles and mobile phones (Aggarwal and McGill 2007; Landwehr et al. 2011).

Most of the academic research has examined the relationship between anthropomorphic product design and psychological processes. For example, the presence of easy-to-anthropomorphize product features (such as faces or specific height relationships) were found to facilitate anthropomorphism by consumers and lead to more favorable evaluations (Aggarwal and McGill 2007). Further, Windhager et al. (2008) demonstrated (by relying upon geometric morphometric techniques) that changes in car designs can result in altered trait associations regarding particular trait dimensions. Other work has shown that whether a product is automatically anthropomorphized by the viewer (or not) depends upon the visual angle of the product (Miesler et al. 2010).

Other recent marketing research has explored specific features of car faces and found a preference for slanted headlights in combination with an upturned mouth (Landwehr et al. 2010), such that the grille influenced perceived friendliness of the car's face, while the headlights influenced both aggressiveness and friendliness. Building upon Russell (1980), it appears that the combination of slanted eyes and an upturned mouth is best, because it equally stimulates pleasure and arousal. The practical value of anthropomorphic product design has also been demonstrated. In particular, products that are easy to anthropomorphize have been shown to lead to more favorable product evaluations (Aggarwal and McGill 2007). Furthermore, a recent study showed that aggressive headlights and a positive design version of the grille had a positive impact on actual car sales (Landwehr et al. 2011).

Similarities between the perception of anthropomorphic products and human characteristics have also been the subject of academic research. In line with expectations, it has been shown that anthropomorphic products embodying unfavorable human characteristics can lead to lower product evaluations (Aggarwal and McGill 2007). Other researchers have concluded that people attributed personality traits to car fronts in a similar fashion as they would to human faces (Windhager et al. 2008). Of relevance to the current research is recent work that has 
found similarities in participant's gaze behavior when visually inspecting anthropomorphic car fronts and human faces (Windhager et al. 2010). Just like when looking at a human face (cf. Armann and Bülthoff 2009), the headlight or eye section of an anthropomorphic car front was found to capture viewers' gazes, which is consistent with the theory of biological preparedness. The researchers interpret their findings as evidence for the existence of a "task independent automaticity of an evolved perceptual bias" that can be elicited by anthropomorphic car fronts.

Although existing research suggests that the perception of faces plays a unique role in human perception with various demonstrated effects on attentional and behavioral tendencies (cf. Gazzaniga et al. 2002), to the best of our knowledge this interplay (particularly with a focus on actually observed behavioral reactions) has not been investigated in the field of anthropomorphic product design. In the next sections, we provide background from related fields in psychology regarding face perception and biological preparedness in terms of product design.

\subsection{Biological preparedness, face perception and product design}

Perception of the human face plays an important role in our day-to-day social interactions, whether it be the recognition of a known face amongst strangers, or the interpretation of facial expressions. The notion that humans possess specialized perceptual mechanisms for faces is confirmed in recent work (cf. Haxby et al. 2002). Not only has research identified particular anatomical structures, such as the fusiform gyrus, that are specialized for human face perception (Kanwisher et al. 1997), it has also collected evidence that these structures are linked to cognitive biases and processing advantages associated with face perception. Underlying mechanisms have been explored in clinical case studies and experiments with subjects suffering from different types of agnosia (Farah et al. 1995; Moscovitch et al. 1997), or in laboratory settings exploring phenomena like the face inversion effect (Ellis 1975; Thompson 1980). To date, there is an on-going discussion as to why these often-observed processing advantages occur for faces (cf. Rousselet et al. 2003).

There appear to be differences in the perception and processing of faces that display different affective states. Studies (usually employing variants of experimental search tasks) suggest that threatening faces are processed more accurately and faster than friendly faces, a finding that has become referred to as the "face in the crowd effect" or more generally the "threat advantage effect" (Fox et al. 2000; Hansen and Hansen 1988; Öhman et al. 2001). These processing differences are not necessarily conscious (Morris et al. 1998) and generally act in accordance with evolutionary perspectives on biological preparedness (Öhman and Dimberg 1978).

Preparedness refers to the phylogenetic "pre-wiring" of certain reactions and automatic behaviors and is important to the biological makeup of an organism. The concept of preparedness is commonly associated with work of Martin Seligman (Seligman 1970), who argued that preparedness can be observed across various species in different learning contexts. Biological preparedness is argued to underlie many psychological processes such as language acquisition, fear acquisition (as in the case of phobias), and the perception of socially relevant stimuli-such as the 
perception of emotional, especially threatening faces (Öhman and Dimberg 1978). Underlying most of the latter work is the notion that advantage exists to organisms that can perceive potentially threatening stimuli, since those organisms (assuming they respond accordingly) are provided an evolutionary advantage in terms of survival.

In the case of preparedness and fear acquisition, there is evidence from spider phobics that threatening stimuli are fixated on more rapidly, yet avoided in the longer term (Rinck and Becker 2006). The evolutionarily motivated explanation is that it makes sense to recognize dangerous animals (or more generally threatening situations) and to quickly avoid them thereafter. At a meta-level, prepared reactions appear to unfold at consecutive cognitive and behavioral steps, namely stimulus detection and the affiliated avoidance reaction. Such reasoning can also be applied to face perception, although to our knowledge a unifying study does not exist that tests these ideas. Separate research traditions on face perception nonetheless have independently examined these predictions.

In terms of avoidance reactions, a recent study has explored the effect of aggressive and happy faces on gaze responses in a simulated a face-staring contest. In particular, Terburg et al. (2011) showed that the speed of gaze aversion from faces displaying anger is moderated by participants' dominance-related reward sensitivity, such that those higher in dominance showed a slower gaze aversion. Similar to a bar fight, that can start with a face-staring contest, looking at someone's face might help to establish a dominance-submission relationship between opponents (cf. Mazur and Booth 1998). By averting one's gaze, this stressful and potentially dangerous situation may be ended all at once, leading to sudden relief of the situational discomfort. In contrast, happy faces can be considered as automatic reward cues and thus lead to appetitive behavior (Winkielman et al. 2005). This reasoning is in accordance with the logic derived from evolutionary theory regarding threatening stimuli.

\subsection{Hypotheses}

In the context of anthropomorphic product designs, we explore whether biological preparedness and associated processes can also be observed with car faces displaying different types of emotional affect. We contend that the highly specialized facial detection system of humans will (at least in part) apply to the perception of objects displaying anthropomorphic faces, such as with automobile car fronts. If this mechanism holds, we would expect to observe the following: First, the perceptual system for affective car faces is expected to be highly efficient, thereby allowing participants to easily identify threatening car fronts from a set of related stimuli. Second, specific to inherited involuntary behavioral responses, the detection mechanism is anticipated to follow a two-step process. Specifically, more attention will be allocated to a threatening object (rather than to a non-threatening one) which from an evolutionary perspective would allow the viewer to prepare and react more quickly to a potentially harmful situation. After recognizing the potential threat, automatically triggered avoidance reactions then help the person to escape the adverse situation. 
While there is general agreement on the evolutionary logic for our expectations, there is no research (to our knowledge) that tests for these reactions in response to viewing anthropomorphic products, particularly car fronts. As such, the current work extends existing knowledge on the perception of anthropomorphic products by showing that viewers express specific liking or disliking for such products and that such anthropomorphic objects can elicit evolved cognitive and behavioral automatisms. Based on the preceding, we offer the following hypotheses:

H1: When confronted in a first contact situation with anthropomorphic product designs, viewers will spend more attention on threatening design features, than on features that do not display such a threat.

H2: When exposed to anthropomorphic product design for longer times, viewers will exhibit an avoidance reaction for threatening design features, than for features that do not display such a threat.

\subsection{Study overview}

The hypotheses were tested in an eye tracking experiment, as eye tracking data is commonly assumed to reflect the distribution of the viewer's visual attention (Duchowski 2007), and has also been used in relevant, previous research (Rinck and Becker 2006; Windhager et al. 2008, 2010). Moreover, eye tracking methodology allows for a more valid measure of human visual attention than classical search tasks, at least in terms of ecological validity. The stimuli, car fronts with anthropomorphic product design features, were developed on the basis of existing literature on schematic faces and pretesting. The eye tracking experiment consisted of two studies using the same set of research participants. To test H1, Study 1 investigated viewers' first contact with anthropomorphic car designs in a dual choice task, where participants had to choose their preferred car design from the stimulus set. In Study 2, participants were presented with only individual car fronts in a staring-contest task tailored to test $\mathrm{H} 2$.

\section{General method}

\subsection{Stimulus material}

The stimulus set was constructed to reflect real-world anthropomorphically designed car fronts and to implement previous findings from the face perception literature. In particular, stimuli construction was heavily guided by Lundqvist et al. $(1999,2004)$ research on affective schematic faces $(1999,2004)$. Although several operationalizations are reported in the literature, we relied upon the highly standardized Lundqvist stimulus set, as a review found it to score highest on threat perception scales and reasonably high on naturalness scales (Horstmann 2009). Several minor modifications (such as combining eyebrows and eyes to the single entity of an auto's headlights) were performed to adapt the model to the design of anthropomorphic car fronts. 
The advantage served by creating stimuli is that perceptual and evaluative differences of the car faces can be clearly attributed to changes in stimulus configuration and not to other differences between the stimuli. In contrast, extant research on anthropomorphic car fronts has thus far relied on less standardized stimuli, such as frontal views of existing car fronts without experimental manipulation (Windhager et al. 2008), or photographs of existing car fronts that have been manipulated either by inverting the cars grille (Aggarwal and McGill 2007) or by asking professional designers to alter the designs to be more "friendly" or "aggressive" (Landwehr et al. 2010).

The stimuli consisted of eight anthropomorphic car fronts that were designed to differ in their affective value (Purucker 2012). The car features (headlights, lower air vent and side air vents) were designed to resemble schematic facial features (respectively, the eye region, the mouth and the cheeks). Each design feature existed in a threatening and a non-threatening variant, resulting in a total set of eight car fronts $(2 \times 2 \times 2)$. An unbranded, graphically altered car body of a brand not available for sale in the participants' country served as the basic car shape. This approach was adopted for the study in order to avoid undesired preference effects that might result from recognizing an existing brand. This approach was also validated in a pre-study. The threatening and non-threatening variants of a particular feature used the same shape but differed in orientation. The stimulus set was validated in a separate study and confirmed to convey the intended affective attributes (Purucker 2012). Therefore, the stimulus set as well as its size closely followed the stimuli of Lundqvist et al. (1999, 2004). Figure 1 depicts two design examples of threatening and non-threatening feature expressions.

In the mentioned research, effects of the headlight and lower air vent designs were found on several affective and liking scales, but not for the side air vents (Purucker 2012). For reasons of ecological validity and theoretical grounding, however, variants of the side air vents were left in the research design. As subsequently detailed, stimuli were presented to participants such that the two phases of the previously described perceptual process correspond to the two reported studies.
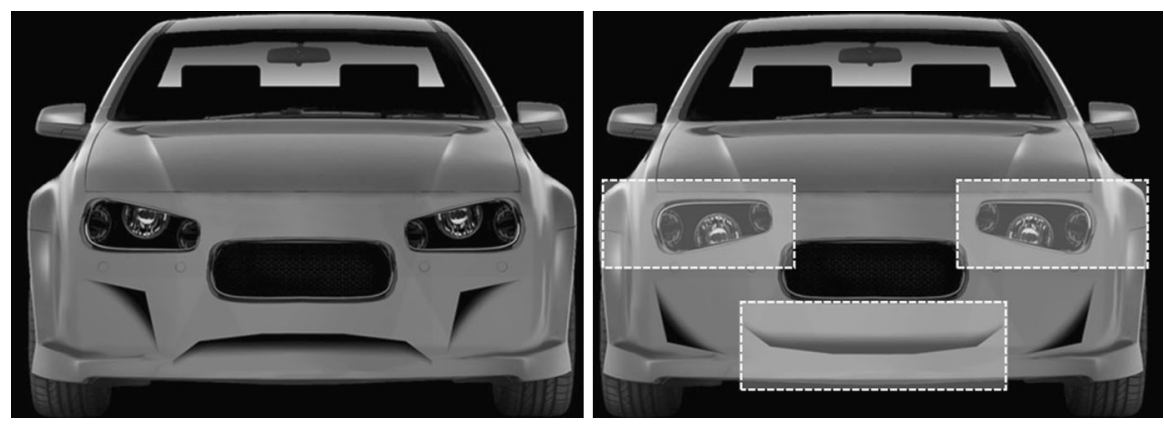

Fig. 1 Example stimuli. Left Car front with the threatening variants for headlights, lower air vent and side air vents. Right Car front consisting of the non-threatening feature variants, with regions of interest drawn as an overlay 


\subsection{Technical implementation}

The experimental procedure was programmed and presented using E-Prime (v2.0.8.22, Psychology Software Tools); data were recorded with Tobii Studio (v2.1.10, Tobii Technology AB), which was also used for basic processing of the eye tracking data. For the collection of eye tracking data, a Tobii X120 standalone eye tracking unit was used. The unit captured data with an accuracy of $0.5^{\circ}$ at a sampling rate of $120 \mathrm{~Hz}$. Eye tracking data for all participants were recorded across the entire stimulus set, with individual trials indicated by trigger signals in the data output. The stimuli were presented on a 19-in. Samsung SyncMaster 913TM monitor with a screen resolution of 1,280 × 1,024 pixels. Participants were seated approximately $55 \mathrm{~cm}$ away from the screen. Head movements were allowed in a box with a side length of roughly $30 \mathrm{~cm}$.

\subsection{Participants}

The sample $(N=39)$ included students and university staff from a large European research university who took part in the study for partial course credit and/or the participation in a raffle. Participant's mean age was $M=26.92$ years $(S D=11.01)$, 13 were females. While all participants initially agreed to participate in all studies of the eye tracking experiment, some participants had to be excluded from some substudy analyses for various reasons, as subsequently detailed.

\section{Study 1}

The aim of Study 1 was to test H1 and explore whether threatening design features in the anthropomorphic car fronts attracted more visual attention than nonthreatening design features (Lundqvist et al. 2004; Rinck and Becker 2006).

\subsection{Trials and procedure}

After seeing a fixation cross for $2 \mathrm{~s}$ at the center of the screen, participants were shown two car fronts left and right from the center position (the specific position of each car front was randomly determined). By pressing buttons with their left and right index fingers, participants indicated their preference for one of the two designs (this is referred to as the choice data). Participants were told to make a decision as soon as possible. Then, pairs of car fronts from the entire stimulus set were presented to participants. Car fronts in a pair complemented each other in all design features. For example, a car with the threatening headlights design was only shown with a car front with the non-threatening headlights. As a result, four pairs of car fronts were shown in a within-subject design, ensuring a reasonably high number of cases for analysis and value estimation (cf. Table 1). Each car was shown twice, once on each side of the screen. The eye tracker was used to track participants' eye movements while making their decisions; this procedure resulted in the eye tracking 
Table 1 Frequencies of car choices from Study 1

\begin{tabular}{|c|c|c|c|c|c|}
\hline \multicolumn{2}{|l|}{ Comparison } & \multicolumn{3}{|l|}{ Choice } & \multirow[t]{2}{*}{$p$} \\
\hline Car front 1 & Car front 1 & $\begin{array}{l}\text { Car front } 1 \\
N(\%)\end{array}$ & $\begin{array}{l}\text { Car front } 1 \\
N(\%)\end{array}$ & $\begin{array}{l}\text { Total } \\
N(\%)\end{array}$ & \\
\hline H-t, SA-t, LA-t & H-n, SA-n, LA-n & $52(67.5)$ & $25(32.5)$ & $77(100.0)$ & .001 \\
\hline H-t, SA-t, LA-n & H-n, SA-n, LA-t & $64(82.1)$ & 14 (17.9) & $78(100.0)$ & .000 \\
\hline H-t, SA-n, LA-t & H-n, SA-t, LA-n & $50(64.1)$ & $28(35.9)$ & $78(100.0)$ & .008 \\
\hline H-t, SA-n, LA-n & H-n, SA-t, LA-t & $56(72.7)$ & $21(27.3)$ & $77(100.0)$ & .000 \\
\hline
\end{tabular}

$H$ headlights, $S A$ side air vents, $L A$ low er air vents; $-t$ threatening design, $-n$ non-threatening design

data reported below. Training was carried out before the experiment to ensure participants understood the procedure.

\subsection{Analysis sample}

Concerning the choice data, none of the participants had to be excluded, as all data were completely recorded. Regarding eye tracking data, however, nine participants were excluded since less than $80 \%$ of their tracking data was properly logged; a situation found frequently in other eye tracking research (cf. Duchowski 2007). As a result, the number of participants was $N=30$ for Study 1 (10 females), with a mean age of $M=25.27$ years $(S D=8.16)$. Considering that no pre-screening was implemented (e.g., not excluding participants with glasses or with their visual acuity below a certain cut-off), the dropout-rate for Study 1 is within an acceptable range.

\subsection{Results}

\subsubsection{Choice data}

Choice results of Study 1 are summarized in Table 1 . Statistically significant choice preferences manifested for all choice pairs $(p<.01)$. Car front designs containing the threatening headlight design were significantly preferred over their opposing designs, as confirmed in a logistic regression $(p<.01)$. While no significant preference tendency could be observed for the side air vents design $(p=.24)$, participants appeared to choose the non-threatening lower air vents design more often $(p=.02)$.

\subsubsection{Eye tracking data}

To analyze the eye tracking data from Study 1, regions of interest were drawn around separate design features, as depicted in Fig. 1. Left and right headlights were conjoined to a single ROI, as they can be considered a single design unit. Eye tracking metrics were calculated, such as the time to first fixation, the first fixation duration and the total fixation duration. The effect of the design feature (e.g., headlights and lower air vent), the affective value of the feature (threatening or non- 
threatening), and their interaction on the eye tracking metrics were modeled with a linear mixed-model approach. This approach accounts for inter-person heterogeneity by specifying a random intercept (Fitzmaurice et al. 2004). Since the choice data did not reveal a clear preference for the side air vent design (and previous studies raised doubts concerning their affective value; cf. Purucker 2012), the side air vents design was not included in this investigation. Separate models were calculated for the different eye tracking metrics and car positions on the screen (left and right). All models were estimated with the lme()-function of the nmle package of the statistic software R (Pinheiro et al. 2008). Parameters for the different models are summarized in Table 2.

The model for time to first fixation for cars shown on the left side of the screen yielded significant coefficients for the lower air vent. In contrast to the headlights (serving as a baseline), the time to first fixation of the lower air vent was significantly prolonged $(b=1.180, S E=0.258, t=4.577, p<.01)$. A significant interaction effect emerged, reporting an even longer time to first fixation for the non-threatening version of the lower air vent design $(b=0.794, S E=0.394$, $t=2.016, p=0.05)$. For car fronts presented on the right side of the screen, only a marginally significant effect of the lower air vent design was found, pointing the same direction $(p<.1)$. No effects were found in the first fixation duration.

Analyzing the total fixation duration, significant effects for the design feature, as well as the interaction between design feature and affective value, manifested for the cars presented on the left half of the screen. The lower air vent was fixated in total for a shorter amount of time $(b=-0.743, S E=0.085, t=-8.782, p<.01)$. The interaction indicated that the non-threatening version of the lower air vent led to longer total fixation durations $(b=0.246, S E=0.120, t=2.049, p<.05)$. An effect of marginal significance was found for the affective value of the design feature, indicating that non-threatening design features were looked at for a shorter period of time $(b=-0.149, S E=0.085, t=-1.749, p<.1)$. For cars presented on the right-hand-side, only a significant effect for the design feature was observed, pointing into the same direction as noted before $(b=-0.444, S E=0.074$, $t=-6.008, p<.01)$.

\subsection{Discussion}

The choice pattern observed in Study 1 generally supports $\mathrm{H} 1$ and confirms findings from previous research (cf. Landwehr et al. 2011; Windhager et al. 2010). Participants clearly preferred cars with threatening headlights and a non-threatening lower air vent design. In accordance with Purucker (2012), the headlight design proved to be the predominant driver of preference in our sample.

Concerning total fixation duration, there was a tendency for less visual attention for non-threatening design features and a significant interaction on the first fixation duration such that the non-threatening mouth was fixated upon later. Contrary to our expectation, there was a significant interaction on the total fixation duration which suggests a longer fixation of the non-threatening mouth-an indication that the effect on this metric primarily results from the affective value of the headlights. Overall, results of Study 1 are supportive of $\mathrm{H} 1$ and an "early" threat advantage 


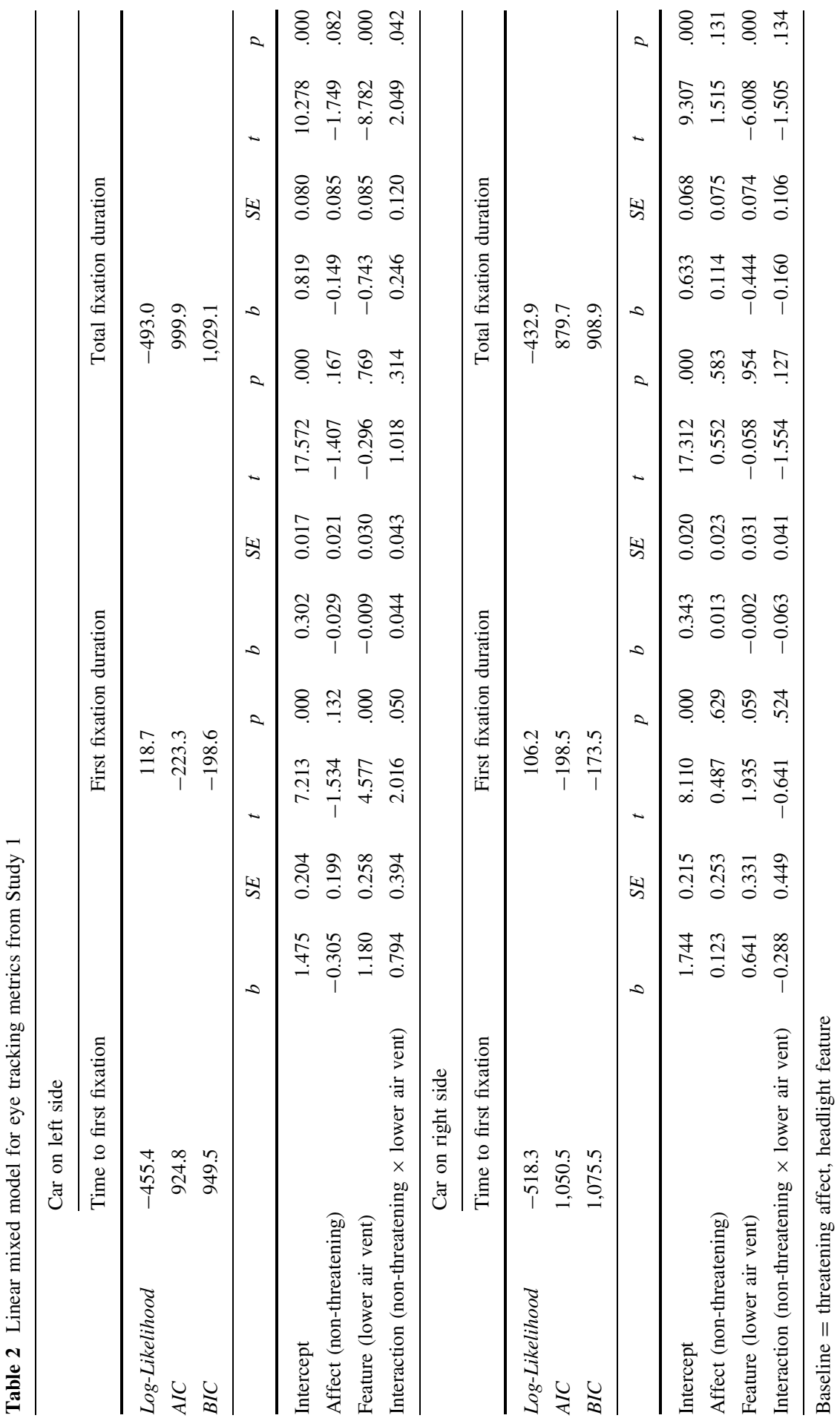


effect, in accordance with what has been previously outlined in the related literature (Öhman et al. 2001; Rinck and Becker 2006).

The effects in Study 1 mainly held for cars presented on the left side of the screen. A possible reason for this lies in a cultural predisposition and the nature of the task employed. Given the typical reading direction for these Western participants, one would expect them to look first to the left, and later to the right. Furthermore, based on the specification of the task (where the exact opposite stimulus was always shown on the other side), an examination of the right-hand stimulus might be somewhat redundant and thus lead to a less intense effort in looking at that stimulus over the course of the study. That said, the results from the eye tracking data in Study 1 clearly support the importance of the headlight region (cf. Windhager et al. 2010), which is fixated faster and more intense in comparison to the lower air vent region, regardless of the affective value of the design.

\section{Study 2}

The goal of Study 2 was to test $\mathrm{H} 2$ by examining visual attention directed towards the affective features of anthropomorphic car fronts in an experimental context resembling a staring contest (cf. Terburg et al. 2011). Based on an evolutionary perspective, participants should look at threatening features less often during longer exposure in order to avoid the threatening stimuli.

\subsection{Trials and procedure}

In a within-subject design, single car images from the picture set were presented to participants in a random order. Each image was shown for $10 \mathrm{~s}$. Preceding, a fixation cross was centered on the position of the car's hood and shown for $2 \mathrm{~s}$. After each picture, participants were asked questions referring to the affective dimensions of the car front. Each picture covered $63 \%$ of the width of the screen and $52 \%$ of the height of the screen, so that the car faces resembled real car fronts seen from a distance of approximately $5 \frac{1}{2} \mathrm{~m}$. Before beginning the experiment, participants were acclimated to the eye tracker and a calibration procedure was carried out. Participants also received written and oral instructions regarding the trials and performed an unrelated test procedure with feedback to ensure their understanding.

\subsection{Analysis sample}

Participants were the same as those in Study 1. One participant had aborted the experiment and was not included in the following analyses. Trials with $20 \%$ or more of the data points missing were dropped; six participants were excluded from the analyses, thus resulting in a final sample of $N=32$ participants, 10 of which were females. The mean age was $M=26.31$ years $(S D=11.21)$. 


\subsection{Results}

\subsubsection{Eye tracking data}

Analysis of Study 2 eye tracking data was conducted in a fashion analogous to that of Study 1. Regions of interest were defined, and linear mixed models with random intercepts were calculated for several eye tracking metrics that served as dependent variables. Design feature (headlights and lower air vent) and affective value (threatening and non-threatening), as well as an interaction of the two were included in the model. Results from different models are summarized in Table 3.

Concerning the time to first fixation, a significant main effect was found for the design feature. In contrast to the headlights region, the lower air vent region was fixated only after a significantly prolonged amount of time $(b=1.823, S E=0.268$, $t=6.789, p<.01)$. No effects were found for the first fixation duration. The model for the total fixation duration yielded significant main effects for affective value and design feature. A largely reduced total fixation duration was observed for the lower air vent region $(b=-2.961, S E=0.151, t=-19.660, p<.01)$. Concerning the effect of the design features, it was found that the non-threatening versions of the design features led to an increased total fixation duration $(b=0.395, S E=0.151$, $t=2.621, p<.01)$. These results provide support for $\mathrm{H} 2$.

\subsection{Discussion}

Study 2's task was designed to resemble a staring contest. Given that participants had seen the entire stimulus set in Study 1, the context of Study 2 clearly simulates a repeated contact situation where one would expect to observe threat avoidance. As expected and in accordance with $\mathrm{H} 2$, design features displaying a threatening feature were fixated for shorter amounts of time, in comparison to non-threatening design features. The data suggested an elevated importance of the headlight region, as this region is fixated faster and longer than the other design features.

\section{General discussion}

In an eye tracking experiment, we investigated the effect of anthropomorphic product design on viewers' perceptions, as measured by choice and visual attention. The aim of the research was to demonstrate how affective design features are able to elicit reactions in customers who are looking at those products, similar to what would be predicted when viewing human faces. Results from the eye tracking studies confirmed processing similarities between anthropomorphic car fronts and human faces (Windhager et al. 2010).

Building from an evolutionary perspective and a phenomenon described as the "threat advantage effect," we hypothesized that design features displaying threatening affect would initially draw viewers' attention, while over the longer term such features would tend to be ignored. The results of our eye tracking studies were largely in line with these predictions. Study 1 simulated a first contact situation 


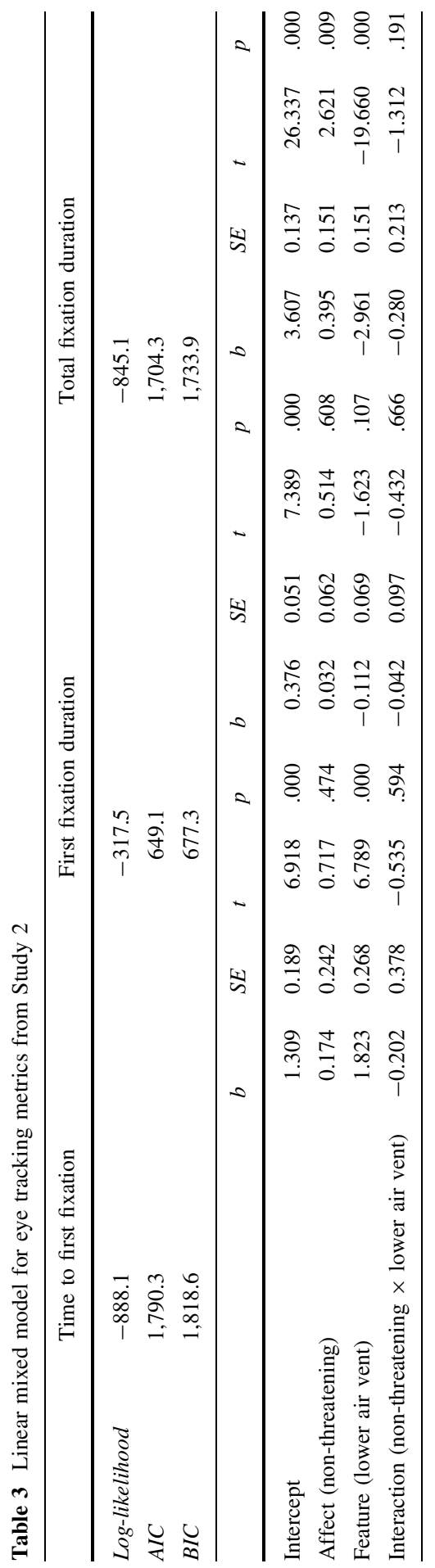


whereby participants were directly confronted with stimuli for a short, selfdetermined amount of time. Results showed that participants devoted more attention to threatening design features, as compared to non-threatening ones. In contrast, participants were forced to look at affectively varied car fronts (resembling a staring contest) in Study 2 with results suggesting that participants tended to avoid threatening features. While the attentional findings support our expectations, choice appears to be independent from attention. Furthermore, as expected from previous research, participants preferred feature combinations of threatening headlights and non-threatening lower air vent for car front designs (Landwehr et al. 2011).

The current studies extend existing research on anthropomorphic product design by suggesting a possible two-step process underlying preference formation. First, there appears to be a pre-attentive, automatic mechanism that is shaped largely by evolutionary influences where people attend to threatening stimuli. The second process, which is more conscious in nature, involves people avoiding stimuli during longer exposures. Such a cognitive mechanism would suggest that other aspects of the product design may be also considered when forming impressions or preferences for a particular product. Indeed, the recent finding that consumers prefer a mixture of threatening headlights and a smiling grille (Landwehr et al. 2011) underscores that conscious associations elicited by anthropomorphic designs are determined not only by automatic evolutionary-shaped mechanisms, but also by cognitive reasoning. Nonetheless, as noted earlier, the current research clearly underscores the importance of evolutionary theory for product design by showing that such mechanisms are at work when responding to certain regions of the anthropomorphic designed object.

\subsection{Practical implications}

Our work contributes to a growing body of literature supporting the relevance of anthropomorphic product designs. In particular, our research shows that visual stimuli can be systematically used in product design to elicit (within certain limits) specific reactions from the viewer of those stimuli. Similar to previous research showing that baby face schematics in product design can increase cuteness ratings of a product (Miesler et al. 2011), our stimuli showed that the use of threatening stimuli can induce particular gaze patterns in customers when viewing the product. These findings suggest real-world applications in product design. For example, designers implementing facial stimuli in their products can reasonably expect viewers to look at the eyes first, with a particular advantage of threatening over nonthreatening features, at least in first (immediate) contact situations. More broadly, evolutionary theory would suggest various ways by which product designers can influence viewers' reactions to their designs. For example, threatening product features might be implemented to systematically draw attention towards a particular product so that it can "stand out from the crowd." Alternately, it might prove useful to increase a product's threat-potential by making it more appealing to a certain customer group. Of course, when relying on those evolutionary stimuli, a product designer cannot be entirely sure whether this makes the product more appealing to 
all customer groups, but can reasonable expect that there will be a reaction by customers via activation of inherited genetic programs in humans.

\subsection{Limitations and future research}

A common critique of prior research regards the use of schematic faces. This criticism relates to whether experimental stimuli are sufficiently ecologically valid to provoke expected reactions from a viewer. In terms of schematic faces, the argument has been made that a constructed face might be too artificial and therefore impact research findings. A similar concern could be levied against the manipulated car fronts used in the current research. Following evolutionary reasoning (Öhman et al. 2001), however, the direct resemblance of a particular stimulus to the biological model may not matter. In particular, the evolution of templates (genetically inherited programs that help humans with the detection and discrimination of relevant stimuli) should allow the detection of specific stimuli, as well as closely resembling stimuli, irrespective of natural variability. For example, while the perception of affective faces is of evolutionary value (Öhman et al. 2001), the evaluation of perceived facial affect does not require a direct match of a particular a face with a particular template, rather, it is a consideration of deviation from competing alternatives. For the same reason, evolutionary inspired design needs to be consistent with genetic templates, but not a direct match with natural occurring stimuli. That said, future research should explore responses to various stimuli in terms anthropomorphic product design. In addition to visual designs, future research could consider other sensory modalities such as the auditory realm and examination of how people respond to speech-like sounds from products. Further, while the current research (and the extant literature) has predominantly focused on car designs, future research could usefully explore other product categories.

\subsection{Conclusion}

Since the automobile's invention, the designs of cars have been jointly influenced by design considerations and functional dimensions as well. From a functional viewpoint, today's car fronts are heavily influenced by drivers' operating needs (e.g., mirrors, windshield and headlights), as well as practical engineering needs such as the cooling of a fuel-combusting engine. While our research and other work demonstrates that drivers favor anthropomorphic designs for automobiles, what might happen if technology drastically changes and eliminates the need for functional aspects of a car, as could happen with the rise of electric cars and front grilles that no longer need to cool a combustion engine? Perhaps anthropomorphism as a design guideline for automobiles, therefore, might disappear as well. The results of our research, however, suggest that even without a functional need, the implementation of anthropomorphic design might provide additional value. And just imagine what automobile designers might be able to do with respect to anthropomorphic car design if the functional dimensions of the automobile had less of a constraining effect on how the product was designed. 


\section{References}

Aggarwal P, McGill AL (2007) Is that car smiling at me? Schema congruity as a basis for evaluating anthropomorphized products. J Consumer Res 34:468-479

Armann R, Bülthoff I (2009) Gaze behavior in face comparison: the role of sex, task and symmetry. Atten Percept Psychophys 71:1107-1126

Bloch PH (1995) Seeking the ideal form: product design and consumer response. J Mark 59:16-29

DiSalvo C, Gemperle F (2003) From seduction to fulfillment: the use of anthropomorphic form in design. In: Proceedings of the 2003 International Conference on Designing Pleasurable Products and Interfaces. ACM, New York, pp 67-72

Duchowski AT (2007) Eye tracking methodology Theory and practice, 2nd edn. Springer, London

Ellis HD (1975) Recognizing faces. Br J Psychol 66:409-426

Epley N, Waytz A, Cacioppo JT (2007) On seeing human: a three-factor theory of anthropomorphism. Psychol Rev 114:864-886

Farah MJ, Wilson KD, Drain HM, Tanaka JR (1995) The inverted face: inversion effect in prosopagnosia: evidence for mandatory, face-specific perceptual mechanisms. Vis R 35:2089-2093

Fitzmaurice G, Laird N, Ware J (2004) Applied longitudinal analysis. Wiley, Hoboken

Fox E, Lester V, Russo R, Bowles RJ, Pichler A, Dutton K (2000) Facial expressions of emotion: are angry faces detected more efficiently? Cog Emot 14:61-92

Gazzaniga MS, Ivry RB, Mangun GR (2002) Cognitive neuroscience. The biology of the mind, 2nd edn. WW Norton and Company, New York

Hansen CH, Hansen RD (1988) Finding the face in the crowd: an anger superiority effect. J Pers Soc Psychol 54:917-924

Haxby JV, Hoffman EA, Gobbini MI (2002) Human neural systems for face recognition and social communication. Biol Psychiatry 51:59-67

Horstmann G (2009) Visual search for schematic affective faces: stability and variability of search slopes with different instances. Cog Emot 23:355-379

Kanwisher N, McDermott J, Chun MM (1997) The fusiform face area: a module in human extrastriate cortex specialized for face perception. J Neurosci 17:4302-4311

Landwehr JR, McGill AL, Herrmann A (2010) The emotional car face: The optimal mix of friendly and aggressive design elements. In: Proceedings of the 39th Annual Conference of the European Marketing Academy (EMAC), EMAC, Kopenhagen

Landwehr JR, McGill AL, Herrmann A (2011) It's got the look: the effect of friendly and aggressive "facial" expressions on product liking and sales. J Mark 75:132-146

Lorenz K (1950) Ganzheit und Teil in der tierischen und menschlichen Gemeinschaft. Studium Generale 3:455-490

Lundqvist D, Esteves F, Öhman A (1999) The face of wrath: critical features for conveying facial threat. Cog Emot 13:691-711

Lundqvist D, Esteves F, Öhman A (2004) The face of wrath: the role of features and configurations in conveying social threat. Cog Emot 18:161-182

Mazur A, Booth A (1998) Testosterone and dominance in men. Behav Brain Sci 21:353-363

Miesler L, Landwehr JR, Herrmann A, McGill AL (2010) Consumer and product face-to-face: antecedents and consequences of spontaneous face-schema activation. Adv Consum Res 37:536-537

Miesler L, Leder H, Herrmann A (2011) Isn't it cute? An evolutionary perspective of baby-schema effects in visual product designs. Int J Design 5:17-30

Morris JS, Öhman A, Dolan RJ (1998) Conscious and unconscious emotional learning in the human amygdala. Nature 393:467-470

Moscovitch M, Winocur G, Behrmann M (1997) What is special about face recognition? Nineteen experiments on a person with visual object agnosia and dyslexia but normal face recognition. J Cog Neurosci 9:555-604

Öhman A, Dimberg U (1978) Facial expressions as conditioned stimuli for electrodermal responses: a case of "preparedness"? J Pers Soc Psychol 36:1251-1258

Öhman A, Lundqvist D, Esteves F (2001) The face in the crowd revisited: a threat advantage with schematic stimuli. J Pers Soc Psychol 80:381-396

Pinheiro J, Bates D, DebRoy S, Sarkar D, The R Core Team (2008) nlme: linear and nonlinear mixed effects models. (Version 3.1-97) 
Purucker C (2012) When do mouths matter? A cross-cultural assessment of consumers' preferences towards anthropomorphic car fronts. In: Proceedings of the 41st Annual Conference of the European Marketing Academy (EMAC), EMAC, Lisbon

Rinck M, Becker ES (2006) Spider fearful individuals attend to threat, then quickly avoid it: evidence from eye movements. J Abnorm Psychol 115:231-238

Rousselet GA, Mace MJM, Fabre-Thorpe M (2003) Is it an animal? Is it a human face? Fast processing in upright and inverted natural scenes. J Vis 3:440-455

Russell JA (1980) A circumplex model of affect. J Pers Soc Psychol 39:1161-1178

Seligman ME (1970) On the generality of laws of learning. Psychol Rev 77:406-418

Terburg D, Hooiveld N, Aarts H, Kenemans JL, van Honk J (2011) Eye tracking unconscious face-to-face confrontations. Psychol Sci 22:314-319

Thompson P (1980) Margaret thatcher: a new illusion. Perception 9:483-484

Tinbergen N (1951) The study of instinct. Clarendon Press, Oxford

Waytz A, Cacioppo JT, Epley N (2010) Who sees human? The stability and importance of individual differences in anthropomorphism. Perspect Psychol Sci 5:219-232

Windhager S, Slice DE, Schaefer K, Oberzaucher E, Thorstensen T, Grammer K (2008) Face to face. The perception of automotive designs. Hum Nat 19:331-346

Windhager S, Hutzler F, Carbon CC, Oberzaucher E, Schaefer K, Thorstensen T, Grammer K (2010) Laying eyes on headlights: eye movements suggest facial features in cars. Coll Antropologicum 34:1075-1080

Winkielman P, Berridge KC, Wilbarger JL (2005) Unconscious affective reactions to masked happy versus angry faces influence consumption behavior and judgments of value. Pers Soc Psychol Bull 31:121-135 